\title{
Cut locus in a Riemannian manifold
}

\author{
Kohei Hatsuse* and Yoshihiro MoRItA*
}

Let $M$ be a complete $n(\geqslant 2)$-dimensional Riemannian manifold. Let $C(p)$ (resp. $Q(p)$ ) be the cut locus (resp. the first conjugate locus) in the tangent space $T_{P} M$ at a point $p \in M$. We put

$$
V_{i}=\left\{v \in C(p) ; \sharp\left(\exp _{p}^{-1}\left(\exp _{p} v\right) \cap C(p)\right)=i+1\right\}
$$

for a positive integer $i$.

Sugahara [3], Durumeric [1] and others investigated a Riemannian manifold $M$ which satisfies a condition

$$
C(p) \cap Q(p)=\varnothing \quad \text { for a point } \quad p,
$$

and they proved many interesting theorems. Some of them are following

TheOrem A ([3]). Assume $M$ satisfies the condition (A) at p. Then $V_{1}$ is open and dense in $C(p)$.

Throrem B ([1]). Assume $M$ is a compact Riemannian manifold which satisfies the condition (A) at $p$. If $d(p) / i(M)<1+f^{-1}(\arccos (-1 / 4))$ then $C(p)=$ $V_{1} \cup V_{2} \cup V_{3}$ and every $V_{i}$ is a submanifold of $M$ with codimension $i$.

In this note, we investigate a complete Riemannian manifold $M$ which satisfies a condition

$$
i(p)<j(p) \quad \text { for a point } p,
$$

where we let $j(p)=\inf \{\|v\| ; v \in Q(p)\}$, and $j(p)=\infty$ if $Q(p)=\varnothing$.

\section{Condition (B) and cut locus.}

We assume hereafter $M$ satisfies the condition (B) at $p$, and assume sectional curvatures $K_{\sigma}$ of $M$ are bounded from below. Let $i(p)$ be the injective radius at $p$, and let $i(M)$ the injective radius of $M$. Then we can choose a Riemannian metric $g$ on $M$ as $i(M)=1$. We denote by $L(\gamma)$ the length of a piecewise smooth curve $\gamma$.

Let $\delta$ be a lower bound for sectional curvatures $K_{\sigma}$. Let $K_{0}=\min \{\delta, 0\}$ and $N\left(K_{0}\right)$ a simply connected complete Riemannian manifold of constant sectional curvature $K_{0}$. We consider a geodesic triangle $a b c$ in $N\left(K_{0}\right)$ which consists of

Received March 14, 1986.

* Department of Mathematics, Ibaraki University, Mito, Ibaraki 310, Japan. 
three minimizing geodesics $\gamma_{a b}, \gamma_{b c}$ and $\gamma_{c a}$, between $a$ and $b, b$ and $c, c$ and $a$ respectively. When $L\left(\gamma_{a b}\right)=L\left(\gamma_{b c}\right)=x+(1 / 2)$ and $L\left(\gamma_{c a}\right)=1$, we put $f(x)=\Varangle b$. It is obvious from the cosine rule in $N\left(K_{0}\right)$ that (i) $f(x)$ is continuous and strictly decreasing on $[0, \infty)$, (ii) $f(0)=\pi$, and (iii) $f(x) \rightarrow 0$ as $x \rightarrow \infty$.

We put $G=\{v \in C(p) ;\|v\|<j(p)\}$. Let $v, v_{1} \in G$ be different two vectors such that $\exp _{p} v=\exp _{p} v_{1}$. Then $\gamma(t)=\exp _{p} t v$ and $\gamma_{1}(t)=\exp _{p} t v_{1}, 0 \leqslant t \leqslant 1$, are minimizing geodesics from $p$ to $\exp _{p} v$. We define a piecewise curve $\gamma \cdot\left(-\gamma_{1}\right)$ by $\gamma \cdot\left(-\gamma_{1}\right)(t)=\gamma(t)$ if $0 \leqslant t \leqslant 1 / 2$, and $\gamma \cdot\left(-\gamma_{1}\right)(t)=\gamma_{1}(2-2 t)$ if $1 / 2 \leqslant t \leqslant 1$.

LEMMA 1. If we put $\|v\| / i(M)=1+x(v)$ then $\Varangle\left(\dot{\gamma}(1), \dot{\gamma}_{1}(1)\right) \geqslant f(x(v))$.

Proof. Let $l=1+x(v)$. Let $d$ be the distance function in $M$. If $d(\gamma(t)$, $\left.\gamma_{1}(t)\right)<1(=i(M))$ on $[0,1]$ then there exist unique minimizing geodesics $\gamma_{t}$ from $\gamma(t)$ to $\gamma_{1}(t)$. This shows that $H(s)=(\gamma \mid[0, s]) \cdot \gamma_{s} \cdot\left(-\left(\gamma_{1} \mid[0, s]\right)\right)$ is a homotopy of $\gamma \cdot\left(-\gamma_{1}\right)$ in a point curve $p$. Since $M$ satisfies the condition (B) at $p$, $\exp _{p}$ has a maximal rank on $\left\{v \in T_{p} M ;\|v\|<l+\varepsilon\right\}$ for a sufficiently small $\varepsilon>0$. Therefore, there exist some $s_{0} \in[0,1]$ such that $L\left(H\left(s_{0}\right)\right) \geqslant 2(l+\varepsilon)$ from Klingenberg's homotopy lemma (cf. [2], 7.1). On the other hand, $L(H(s)) \leqslant 2 l$ for any $s$, which is a contradiction.

If $d\left(\gamma\left(t^{\prime}\right), \quad \gamma_{1}\left(t^{\prime}\right)\right)=1$ then $l-t^{\prime} l \geqslant l-(1 / 2)=(1 / 2)+x(v)$. Therefore, Lemma follows from Toponogov's comparison theorem.

We put $h(v)=\sharp\left(\exp _{p}^{-1}\left(\exp _{p} v\right) \cap C(p)\right)$ for $v \in G$, and $W_{i}=V_{i} \cap G$. Then $W_{i}=$ $h^{-1}(i+1)$.

LeMmA 2 ([3], Lemma 3.6). If $h$ is constant in a neighborhood $U$ in $G$ then $U \subset W_{1}$.

THEOREM 1. Assume $M$ is a complete Riemannian manifold which satisfies the conditon (B) at $p$, and assume $K_{\sigma} \geqslant \delta$. Then

(1) $W_{1}$ is open and dense in $G$,

(2) if we put $\theta(v)=f(x(v)) / 2$ then

$$
h(v) \cdot \int_{0}^{\theta(v)} \sin ^{n-2} \theta d \theta \leqslant a
$$

where $a=\pi$ if $n$ is even, and $a=2$ if $n$ is odd.

Proof. Since $h$ is upper semi-continious in $G, W_{1}$ is an open subset of $G$. Let $U$ be a neighberhood of $v \in G$. Then, by the definition of $h, h$ has a minimal value at $w \in U$, and hence $h$ is locally constant around $w$. Therefore Lemma 2 shows that $W_{1}$ is dense in $G$.

Let $q=\exp _{p} v$. If $h(v)=k$ then $\exp _{p}^{-1}\left(\exp _{p} v\right) \cap C(p)=\left\{v, v_{2}, \ldots, v_{k}\right\}$. Let $\gamma_{i}(t)=\exp _{p} t v_{i}, 0 \leqslant t \leqslant 1$. Then $q$ is a cut point along every geodesic $\gamma_{i}$. Therefore $\left\|v_{i}\right\|=\|v\|$ and hence $x\left(v_{i}\right)=x(v)$. If we put $v_{1}=v$ then Lemma 1 shows that 


$$
\Varangle\left(\dot{\gamma}_{i}(1), \dot{\gamma}_{j}(1)\right) \geqslant f(x(v)) \quad 1 \leqslant i<j \leqslant k .
$$

Let $u_{i}=\dot{\gamma}_{i}(1) /\left\|\dot{\gamma}_{i}(1)\right\|$. Let $S$ be a unit sphere centered at the origin in $T_{q} M$. Then $B_{i}=\left\{u \in S ; \Varangle\left(u, u_{i}\right)<f(x(v)) / 2\right\}$ is a neighberhood of $u_{i}$ in $S$, and $B_{i} \cap B_{j}=\varnothing$ if $i \neq j$. Since $\operatorname{vol}\left(\bar{B}_{i}\right)=\operatorname{vol}\left(\bar{B}_{j}\right)$ for any $i, j$, we have $k \cdot \operatorname{vol}\left(\bar{B}_{1}\right) \leqslant \operatorname{vol}(S)$, where $\bar{B}_{i}$ denotes the closure of $B_{i}$. If we put $\theta(v)=f(x(v)) / 2$ then

$$
\operatorname{vol}\left(\bar{B}_{1}\right)=2 \pi^{\frac{n-1}{2}} \cdot \int_{0}^{\theta(v)} \sin ^{n-2} \theta d \theta / \Gamma((n-1) / 2),
$$

where $\Gamma(s)$ is the gamma function. Since $\operatorname{vol}(S)=2 \pi^{\frac{n}{2}} / \Gamma(n / 2)$, we have

$$
k \cdot \int_{0}^{\theta(v)} \sin ^{n-2} \theta d \theta \leqslant \sqrt{\pi} \cdot \Gamma((n-1) / 2) / \Gamma(n / 2) \leqslant a .
$$

Thus we have proved Theorem.

More precisely, Durumeric proved

LemMA $3([1]$, Lemma 3). If $f(x(v)) \leqslant \arccos (-1 / m)$ then $h(v) \leqslant m+1$.

2. Case $f(x(v))>\pi / 2$.

LeMmA 4. Let $w_{1}, w_{2}, \ldots, w_{k}$ be non-zero vectors in $T_{q} M$. If $\Varangle\left(w_{i}, w_{j}\right)>\pi / 2$ for $i<j$ then $w_{1}-w_{k}, w_{2}-w_{k}, \ldots, w_{k-1}-w_{k}$ are linearly independent.

Proof. Lemma is obious for $k=2$. Let $k>2$. We assume Lemma is true for any $k-1$ vectors of $w_{1}, w_{2}, \ldots, w_{k}$. If Lemma is false for $k$ then

$$
w_{1}-w_{k}=\sum_{i=2}^{k-1} a_{i}\left(w_{i}-w_{k}\right)
$$

and $a_{i} \neq 0$ for any $i$. Therefore, we can write

$$
w_{1}=\sum_{i=2}^{k} a_{i} w_{i}, \quad \sum_{i=2}^{k} a_{i}=1
$$

and hence $\sum_{i=2}^{k} a_{i}\left(\left\langle w_{1}, w_{1}\right\rangle-\left\langle w_{i}, w_{1}\right\rangle\right)=0$. This shows that some of $a_{i}$ are positive and others are negative. We assume $a_{2}, \ldots, a_{l}$ are positive and $a_{l+1}, \ldots, a_{k}$ are negative. If we put $z=\sum_{i=2}^{b} a_{i} w_{i}$ then $\left\langle z, w_{i}\right\rangle<0$ for any $i$. Therefore, we have

$$
0 \leqslant\langle z, z\rangle=\sum_{i=2}^{l} a_{i}\left\langle z, w_{i}\right\rangle<0
$$

Thus we have proved Lemma.

THEOREM 2. Assume $M$ is a complete Riemannian manifold which satisfies the condition (B) at $p$, and assume $K_{\sigma} \geqslant \delta$. If $f(x(v))>\pi / 2$ for any $v \in G$ then $G=W_{1} \cup W_{2} \cup \cdots \cup W_{n}$ and every $W_{i}$ is a submanifold of $M$ with codimension $i$. 
Proof. We fix a vector $v_{1} \in G$. Let $\exp _{p}^{-1}\left(\exp _{p} v_{1}\right) \cap C(p)=\left\{v_{1}, v_{2}, \ldots, v_{k}\right\}$. Let $\gamma_{i}(t)=\exp _{p} t v_{i}, 0 \leqslant t \leqslant 1$. Then Lemma 1 shows that $\Varangle\left(\dot{\gamma}_{i}(1), \dot{\gamma}_{j}(1)\right) \geqslant f(x(v))>$ $\pi / 2$ for $i<j$. Therefore, Lemma 4 shows that $k \leqslant n+1$. Since $W_{i}=h^{-1}(i+1)$, we have $G=W_{1} \cup W_{2} \cup \cdots \cup W_{n}$.

Let $l=\left\|v_{1}\right\|$ and $B=\left\{u \in T_{p} M ;\|u\| \leqslant l+\varepsilon\right\}$ for $\varepsilon>0$. Let $h_{1}(u)=$ $\#\left(\exp _{p}^{-1}\left(\exp _{p} u\right) \cap B\right)$ for $u \in B$. Then $1 \leqslant h_{1}(u)<\infty$ and $h_{1}(u)$ is upper semicontinuous on $B$. If we choose $\varepsilon>0$ sufficiently small then we have $h_{1}\left(v_{1}\right)=$ $h\left(v_{1}\right)$. Since $l<j(p)$, there exists a neighborhood $U_{i}$ of $v_{i}$ such that (i) $h_{1}(u) \leqslant k$ in $U_{1}$, and (ii) $\exp _{p} \mid U_{i}$ is diffeomorphic and $\exp _{p} U_{i}=\exp _{p} U_{1}$. For $u_{1} \in U_{1}$, there exists from (ii) unique $u_{i} \in U_{i}$, and $\exp _{p} u_{i}=\exp _{p} u_{1}$. This shows that $h_{1}(u)$ is constant $k$ in $U_{1}$. On the other hand, we can find a minimizing geodesic $\exp _{p} t u_{0}$, $0 \leqslant t \leqslant 1$, from $p$ to $\exp _{p} u_{1}$. Since $h_{1}\left(u_{1}\right)=k$, we have $u_{0}=u_{i}$ for some $i$. Therefore $u_{1} \in C(p)$ if $\left\|u_{i}\right\|=\left\|u_{1}\right\|$ for any $i$. If we define a mapping $\varphi: U_{1} \rightarrow R^{k-1}$ by $\varphi\left(u_{1}\right)=\left(\left\|u_{1}\right\|^{2}-\left\|u_{k}\right\|^{2}, \ldots,\left\|u_{k-1}\right\|^{2}-\left\|u_{k}\right\|^{2}\right)$ then Lemma 4 shows that $\varphi$ has rank $k-1$ at $v_{1}$. Thus we have proved Theorem.

When $M$ is compact, we put $d(p)=\sup \{\|v\| ; v \in C(p)\}$.

COROllary. Assume $M$ is a compact Riemannian manifold which satisfies the condition (A) at $p$. If $d(p) / i(M)<1+f^{-1}(\pi / 2)$ then $C(p)=V_{1} \cup V_{2} \cup \cdots \cup V_{n}$ and every $V_{i}$ is a submanifold of $M$ with codimension $i$.

From Lemma 3, we can prove following

THEOREM 3. Assume $M$ is a complete Riemannian manifold which satisfies the condition (B) at $p$, and assume $K_{\sigma} \geqslant \delta$. If $f(x(v)) \geqslant \arccos (-1 / m)$ then $G=$ $W_{1} \cup W_{2} \cup \cdots \cup W_{m}$.

COROLlary. Assume $M$ is a compact Riemannian manifold which satisfies the condition (A) at $p$. If $d(p) / i(M) \leqslant 1+f^{-1}(\arccos (-1 / m))$ then $C(p)=V_{1} \mathrm{U}$ $V_{2} \cup \cdots \cup V_{m}$.

\section{References}

[1] O. Durumeric, Manifolds with almost equal diameter and injectivity radius, J. Differential Geom., 19 (1984), 453-474.

[2] D. Gromoll, W. Klingenberg and W. Meyer, Rimannsche Geometrie im Grossen, Lecture notes in Math. vol. 55, Springer, Berlin, 1968.

[3] K. Sugahara, On the cut locus and the topology of Riemannian manifolds, J. Math. Kyoto Univ., 14 (1974), 391-411. 